\title{
Updated Results of High-Dose Rate Brachytherapy and External Beam Radiotherapy for Locally and Locally Advanced Prostate Cancer Using the RTOG-ASTRO Phoenix Definition
}

\author{
Antonio C. Pellizzon, Joao Salvajoli, Paulo Novaes, Maria Maia, Ricardo Fogaroli
}

Department of Radiation Oncology, AC Camargo Hospital, Sao Paulo, SP, Brazil

\begin{abstract}
Purpose: To evaluate the prognostic factors for patients with local or locally advanced prostate cancer treated with external beam radiotherapy (RT) and high dose rate brachytherapy (HDR) according to the RTOG-ASTRO Phoenix Consensus Conference.

Materials and Methods: The charts of 209 patients treated between 1997 and 2005 with localized RT and HDR as a boost at the Department of Radiation Oncology, AC Camargo Hospital, Sao Paulo, Brazil were reviewed. Clinical and treatment parameters i.e.: patient's age, Gleason score, clinical stage, initial PSA (iPSA), risk group (RG) for biochemical failure, doses of RT and HDR were evaluated. Median age and median follow-up time were 68 and 5.3 years, respectively. Median RT and HDR doses were 45 Gy and 20 Gy.

Results: Disease specific survival (DSS) at 3.3 year was 94.2\%. Regarding RG, for the LR (low risk), IR (intermediate risk) and HR (high risk), the DSS rates at 3.3 years were $91.5 \%, 90.2 \%$ and $88.5 \%$, respectively. On univariate analysis prognostic factors related to DSS were RG $(\mathrm{p}=0.040)$, Gleason score $\leq 6 \mathrm{ng} / \mathrm{mL}(\mathrm{p}=0.002)$, total dose of HDR $\geq 20$ Gy $(\mathrm{p}<0.001)$ On multivariate analysis the only statistical significant predictive factor for biochemical control (bNED) was the RG, p $<0.001$ (CI - 1.147-3.561).

Conclusions: Although the radiation dose administered to the prostate is an important factor related to bNED, this could not be established with statistical significance in this group of patients. To date, in our own experience, HDR associated to RT could be considered a successful approach in the treatment of prostate cancer.
\end{abstract}

Key words: prostate cancer; radiotherapy; brachytherapy; recurrence; treatment failure

Int Braz J Urol. 2008; 34: 293-301

\section{INTRODUCTION}

Prostate cancer $(\mathrm{PCa})$ is one of the most prevalent malignancies affecting men in the developed world. For male population of western countries, the probability of deaths from PCa is about $3 \%$ (1). In Brazil for 2006, 47,280 new cases of PCa were expected to be diagnosed (2). The optimal management of both localized and locally advanced PCa remains contro- versial with a consensus that surgery, radiotherapy, hormonal therapy, and watchful waiting can be used isolated or in combination to treat the different risk groups (RG) for biochemical failure (BF) (3).

Radiotherapy (RT) has been used for years to treat patients with poor clinical conditions or with advanced stages of the disease, but in the past two decades it has emerged as an option to surgery in some specific cases. For patients treated with RT 
significant clinical data are available demonstrating that patients have a significantly better outcome as the dose administered to the prostate is increased (4-6). There are also a number of published results demonstrating that conformal high dose rate (HDR) brachytherapy is a successful method for delivering higher dose of radiation to the prostate sparing the normal surrounding tissues $(7,8)$. Indeed, HDR is a very precise and conformal way of dose delivery with cell killing effects comparable to three-dimensional conformal (3DRT) and intensity modulated (IMRT) external beam RT (9). HDR also has some potential additional advantages over normal tissue sparing and on reducing miss dose to the prostate, due imprecise target localization, treatment setup uncertainties, organ motion and or deformation during the treatments, with a relative low incidence of severe acute and late side-effects (10-12).

\section{MATERIAL AND METHODS}

This study on human beings has been approved by the local Ethics Committee of Hospital AC Camargo, Sao Paulo, Brazil. The charts of patients with initial or locally advanced biopsy proven prostate adenocarcinoma, treated with pelvic localized conventional or 3DRT in combination with HDR at the Department of Radiation Oncology, Hospital AC Camargo, Sao Paulo, Brazil were retrospectively reviewed. Details such as Gleason score (GS), the initial PSA value (iPSA) and clinical stage (CS) using the 1992 AJCC clinical stage were collected from the hospital records to define the risk group for biochemical failure, according to the following classification: low risk subgroup (LR) encompassed patients with CS T2a or lesser, GS less than 7 and initial PSA value equal or lesser than $10 \mathrm{ng} / \mathrm{mL}$. Patients with either stage T2b, GS 7 or initial PSA value ranging from $10-20 \mathrm{ng} / \mathrm{mL}$ were considered intermediate risk (IR) and the remaining patients or patients who presented two or more of the characteristics of the IR subgroup were grouped into the high risk (HR) group for BF (13). At the discretion of the referral urologists, patients in all groups received a course of neoadjuvant central or peripheral androgen blockage (NAAD), with goserelin and or flutamide or ciproteron acetate, 3 to 6 months prior to RT.

In the first three years of treatment, conventional (2D) RT planning was used for all patients, based on diagnostic computed tomography scan (CT), urethrogram and rectal contrast agent to assist in defining prostate, seminal vesicles and normal tissue volumes at risk. The prostate and seminal vesicles were irradiated $t$ via a $6 \mathrm{MV}$ Varian linear accelerator, with four-field box technique and individual protections with cerrobend blocks. After 1999, all patients were treated with localized three-dimensional conformal (3D) RT.

The technique of HDR has been previously reported in the literature (9). In brief, the implant procedures were performed 10 to 15 days after the end of RT, under spinal anesthesia with the patient in lithotomic position. Two metallic markers were inserted into the gland, one in the apex and the other at the base of the prostate, to ascertain quality control of any needle displacement during the treatment and to allow corrections whenever it was necessary. All the implants were performed with steel needles inserted through a perineal template, which was sutured to the perineum. The needles were uniformly placed into the entire prostatic volume, but avoiding the urethra. In the initial years, orthogonal X-rays were used for planning and dosimetric calculations. On August 2000, we switched to 3D image guided CT based planning using the BrachyVision ${ }^{\circledR}$ Planning System (Varian Medical Systems, Inc., Palo Alto CA., USA)

After completion of treatment, patients were seen in follow-up 1 month later and subsequently every 2-4 months for the first 24 months. Thereafter patients were seen at follow-up every 6-12 months.

The endpoint was to evaluate the biological non evidence of disease (bNED), calculated as the interval from pathologic diagnosis of PCa to BF. Date of $\mathrm{BF}$, a rise in $2 \mathrm{ng} / \mathrm{mL}$ after the nadir had been reached, was defined according to the RTOG-ASTRO Phoenix Consensus Conference (14). Pearson chi-square and Student's-t tests were used to compare differences in categorical and continuous patient characteristics, respectively. Survival data were generated using the Kaplan-Meier method, with log-rank test used to compare equality of survivor functions. The Cox proportional hazard model was used for multivariate 
analysis, using a stepwise procedure. Statistical tests were performed using SPSS 13.0 (SPSS, Chicago, IL).

\section{RESULTS}

A total of 234 patients were treated with combination of RT and HDR between 1997 and 2005 at the Department of Radiation Oncology, Hospital AC Camargo, Sao Paulo, Brazil. Fifteen patients were excluded from the study because they were lost to follow-up after treatment. Seven patients had whole pelvis RT and were also excluded from the analysis. Three patients did not reach a nadir value below 10 $\mathrm{ng} / \mathrm{mL}$ after the end of treatment and a review of their charts showed that they already had metastatic disease at the time of treatment, and therefore also excluded from the study. Median age of the remaining 209 patients was 68 years (range, 47-83 years). Median follow-up was 5.3 years (range, 2-10). There were 77 $(36.8 \%)$ of patients in the LR, $65(31.1 \%)$ in the IR group and $67(32.1 \%)$ in the HR group. The patients were well balanced into all groups, with no statistical significant difference in between the different $R G$ for $\mathrm{BF}(\mathrm{p}=0.437)$. Characteristics of patients are shown in Table-1.

The dose of RT ranged from 36 to 54 Gy (median $45 \mathrm{~Gy}$ ) given in 20 to 30 daily fractions of 1.8 or $2.0 \mathrm{~Gy}$. The total RT treatment time ranged from 4 to 7 weeks (median 5 weeks). The HDR was performed after one to two weeks of the completion of RT in 204 patients. Five patients started their treatment by HDR and after that had a course of RT. The total treatment time ranged from 5 to 9 weeks (median 7 weeks). The dose of HDR ranged from 16 to 24 Gy given in 4 fractions, BID in two days. Median HDR dose was 20 Gy.

A hundred and eight (51.7\%) patients had no NAAD. Of the patients who had NAAD, $70(33.5 \%)$ had central and $31(14.8 \%)$ patients had peripheral blockage alone.

Overall survival (OS) rate at 3.3 years was 97.8\%. The 5- and 10-year actuarial OS rates were $95.7 \%$ and $90.6 \%$, respectively (Figure-1). Eleven patients had died at the time of this analysis. Five (2.4\%) patients died due prostate cancer disease progression
Table 1 - Patients characteristics.

\begin{tabular}{lrrrr}
\hline Variables & N & \% & Range & Median \\
\hline Age (years) & & & $47-83$ & 68.0 \\
iPSA (ng/mL) & & & $4-175$ & 10.5 \\
Follow-up & & & $24-120$ & 62.9 \\
$\quad$ (months) & & & & \\
Ethnicity & & & & \\
$\quad$ Asiatic & 8 & 3.8 & & \\
$\quad$ Caucasian & 180 & 86.2 & & \\
$\quad$ African & 14 & 6.7 & & \\
$\quad$ Unknown & 7 & 3.3 & & \\
Clinical stage & & & & \\
$\quad$ T1 & 95 & 45.5 & & \\
$\quad$ T2a & 72 & 34.4 & & \\
$\quad$ T2b & 19 & 9.1 & & \\
$\quad$ T3a & 23 & 11.0 & & \\
Gleason score & & & & \\
$\quad \leq 6$ & 148 & 70.8 & & \\
$\quad 7$ & 41 & 19.6 & & \\
$\quad \geq 8$ & 20 & 9.6 & & \\
RG & & & \\
$\quad$ Low & 77 & 36.8 & \\
Intermediate & 65 & 31.2 & \\
$\quad$ High & 67 & 32.2 & \\
NAAD & & & \\
$\quad$ No & 108 & 51.7 & \\
$\quad$ Yes & 101 & 48.3 & \\
Total & 209 & 100.0 & & \\
\hline
\end{tabular}

iPSA = initial PSA value; $N A A D=$ neoadjuvant androgen deprivation; $R G=$ risk group for biochemical failure.

and $6(2.9 \%)$ of other causes ( 3 of cardiologic related disease, 2 of a new second primary tumor and 1 of surgical complication after a salvage prostatectomy). For the LR, IR and HR, the bNED rates at 3.3 years were $91.5 \%, 90.2 \%$ and $88.5 \%$, respectively. Actuarial bNED rates at 5- and 10 -year were $95.7 \%$ and $59.6 \%$, as shown in Figure-2.

On univariate analysis the prognostic factors related to better bNED rates were $\mathrm{RG}(\mathrm{p}=0.040)$, GS $\leq 6 \mathrm{ng} / \mathrm{mL}(\mathrm{p}=0.002)$, total dose of HDR $\geq 20$ Gy (p $<0.001$ ), use of three dimensional planning for HDR 


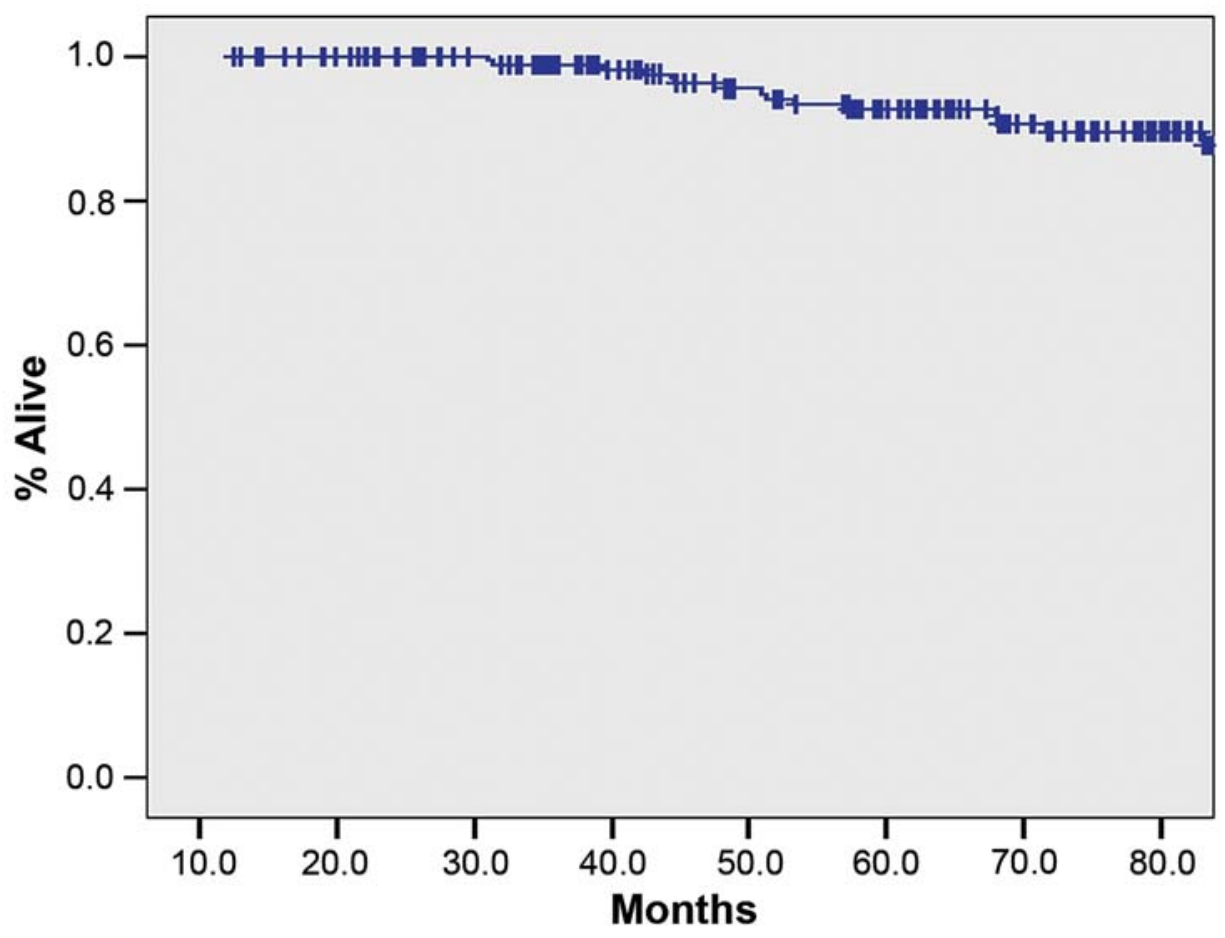

$\begin{array}{lcccccccc}\text { At Risk 209 } & 181 & 154 & 149 & 133 & 117 & 85 & 59 & \\ (\%) & 100 & 86.6 & 73.6 & 71.3 & 63.6 & 56.0 & 40.7 & 28.2\end{array}$

Figure 1 - Overall survival.

$(\mathrm{p}<0.001)$ Table-2. We also explored the influence of NAAD according to $\mathrm{RG}$, observing no statically significant benefit of association of NAAD in any group $(\mathrm{p}=0.061)$.

On multivariate analysis, the only statistical significant predictive factor related to a better bNED rate was the $\mathrm{RG}, \mathrm{p}<0.001$ (CI - 1.147-3.561). The hazard ratio was 1.7 and 2.4 times less chance of achieving a bNED for IR and HR when compared to LR, respectively, (Figure-3).

\section{COMMENTS}

Several retrospective studies with more than 5 -year follow up have previously described the outcome of patients treated with combination of RT and
$\operatorname{HDR}(8,14,15)$, but data from prospective randomized trail comparing results of this combination with dose escalation RT3D or IMRT is still missing.

The main advantage of HDR is its ability to deliver a relative high dose of radiation within a well-defined volume, with a rapid fall-off of dose outside the implanted area. This approach is ideal for the treatment of prostate cancer, where the gland lays very close to critical normal tissues, in particular the anterior rectal wall and bladder neck (9).

Galalae et al. (16) also investigated the long-term outcome by RG using HDR and RT with or without NAAD. There were 611 patients grouped as follows: 46 patients into LR, 188 patients into IR and 359 patients considered HR. Using the ASTRO definition for BF these authors observed that the actuarial bNED and disease free survival rates at 


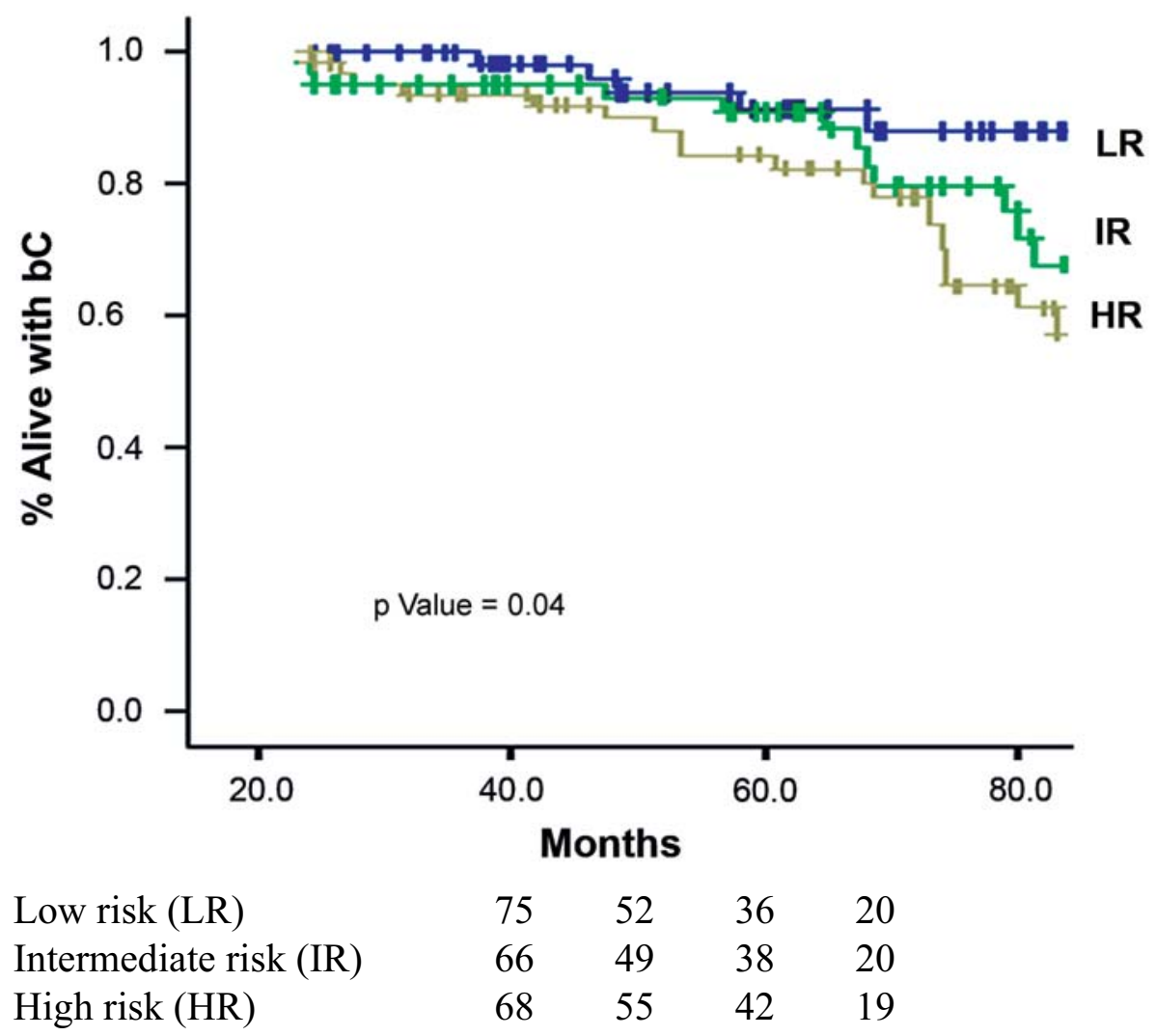

Figure 2-Biochemical control according to the risk group for biochemical failure.

5-year and 10 -year were $77 \%, 67 \%, 73 \%$ and $49 \%$, respectively. For the different RG the actuarial 5-year bNED rates were $96 \%$ for LR, $88 \%$ for IR and $69 \%$ for HR. They observed that GS and RG were statistical significant predictive factors of bNED, which were also confirmed in the present study, and GS ( $p=0.002)$ and group risk $(\mathrm{p}=0.050)$ were statistical significant predictive factors for BF. They also observed that CS and iPSA were also statistical significant predictive factors, which was not confirmed in this study.

Deger et al. (17) evaluated 422 patients with localized prostate cancer treated between 1992 and 2001 with HDR and 3DRT. All patients underwent laparoscopic pelvic lymph node dissection to exclude patients with lymphatic involvement. The BF was also defined according to the ASTRO criteria. The bNED according to $\mathrm{RG}$ were $100 \% \mathrm{~T} 1,75 \%$ for $\mathrm{T} 2$ and $60 \%$ $\mathrm{T} 3$ at 5 years. Five-year bNED were $81 \%$ in the LR, $65 \%$ in the IR and $59 \%$ in the HR. Five-year OS and bNED were $87 \%$ and $94 \%$, respectively. They also observed that iPSA, RG and age were significantly related to bNED. In our study the use of NAAD was not associated with better bNED $(\mathrm{p}=0.425)$. When the subgroups according to risk for BF were analyzed, there was not a statistical significance for $a$ better bNED related to the use or not of NAAD ( $p$ $=0.875$ ).

There are a scarce number of papers that have published the results of HDR and RT for PCa using the RTOG-ASTRO Phoenix definition. This definition states that "To avoid the artifacts resulting from short follow-up, the reported date of control should be 
Table 2 - Univariate analysis.

\begin{tabular}{|c|c|c|c|c|}
\hline Variables & Total & bNED & $\%$ & p Value* \\
\hline \multicolumn{5}{|l|}{ Age } \\
\hline$<60$ & 25 & 20 & 80.0 & \multirow{3}{*}{0.777} \\
\hline $61-70$ & 88 & 70 & 79.5 & \\
\hline$>70$ & 96 & 83 & 86.5 & \\
\hline \multicolumn{5}{|l|}{ iPSA } \\
\hline$\leq 10$ & 106 & 92 & 86.8 & \multirow{3}{*}{0.788} \\
\hline $10-20$ & 61 & 49 & 80.3 & \\
\hline$>20$ & 42 & 32 & 76.2 & \\
\hline \multicolumn{5}{|l|}{ RG } \\
\hline LR & 77 & 72 & 93.5 & \multirow{3}{*}{0.040} \\
\hline IR & 65 & 53 & 81.5 & \\
\hline HR & 67 & 48 & 71.6 & \\
\hline \multicolumn{5}{|c|}{ Gleason score } \\
\hline$\leq 6$ & 148 & 128 & 86.5 & \multirow{3}{*}{0.002} \\
\hline 7 & 41 & 32 & 78.0 & \\
\hline$\geq 8$ & 20 & 13 & 65.0 & \\
\hline \multicolumn{5}{|l|}{ NAAD } \\
\hline Yes & 106 & 90 & 84.9 & \multirow[b]{2}{*}{0.425} \\
\hline No & 103 & 83 & 80.6 & \\
\hline \multicolumn{5}{|c|}{ HDR-BT (Gy) } \\
\hline$<20$ & 114 & 91 & 79.8 & \multirow{2}{*}{$<0.001$} \\
\hline$\geq 20$ & 95 & 82 & 86.3 & \\
\hline \multicolumn{5}{|c|}{ EBRT (Gy) } \\
\hline$<50$ & 133 & 110 & 82.7 & \multirow{2}{*}{0.324} \\
\hline$\geq 50$ & 76 & 63 & 82.9 & \\
\hline \multicolumn{5}{|l|}{ 3DHDR } \\
\hline No & 147 & 120 & 81.6 & \multirow{2}{*}{$<0.001$} \\
\hline Yes & 62 & 53 & 85.5 & \\
\hline
\end{tabular}

bNED = biochemical control; iPSA = initial PSA value; $R G=$ risk group for biochemical failure; $L R=$ low risk; $I R=$ intermediate risk; $H R=$ high risk; $N A A D=$ neoadjuvant androgen deprivation; $H D R-B T=$ high dose rate brachytherapy; $E B R T$ = external beam radiotherapy; $B E D=$ biological effective dose; $3 D H D R=$ high dose rate brachytherapy based on three dimensional planning. $p^{*}=$ test equality of survival distribution, 95\% confidence interval.

listed as 2 years short of the median follow-up" (14). Chin et al. (18) related the results of 65 consecutive patients treated between 1998 and 2004 with combination of RT and HDR given in 2 fractions. Sixty patients $(92.3 \%)$ were considered IR or HR. With a median follow-up of 3.5 years (range 0.6-5.8), two patients had died of metastatic disease and other four patients had BF, giving a 3-year actuarial bNED rate of $90.8 \%$. Yamada et al. (19) also reported the results of 105 patients consecutively treated between 1998 and 2004 with RT (45-50.4 Gy) and HDR (5.5-7.0 Gy per fraction). With a median follow-up of 44 months (8-79 months), the actuarial 5-year bNED rates for LR, IR and HR were $100 \%, 98 \%$, and $92 \%$, respectively. In the current study the actuarial bNED rates at 5-year were $91.8 \%$ for LR group, $79.3 \%$ for IR group and $69.1 \%$ for HR group $(p=0.040)$, respectively.

Patients considered HR have more chances of BF. This phenomenon could be a consequence of current inadequate imaging of lymph node or bone metastasis or due subclinical metastatic spread that remains undetectable during radical treatment. However, tumor biology itself could lead to the progression of the disease in the HR group. As a consequence, risk-adapted therapy is very important in these cases. The combination of RT and HDR is an alternative strategy of dose escalation that can potentially achieve a better bNED, but for patients at HR the localized dose delivered by HDR may be a potential disadvantage, because a microscopic spread outside the prostate and even its capsule may occur. In these cases, the combination of HDR and RT can provide treatment to potential areas of microscopic spread. What is still not answered is if adding pelvic radiation, instead to localized RT in combination to HDR for PCa patients with a more than $15 \%$ risk of positive lymph nodes will really improve outcome.

The use of NAAD still remains controversial for patients with IR to HR. Martinez et al. (20) in a study of 1,260 patients treated with pelvic RT and HDR observed similar OS, DFS and bNED for patients who were treated with or without the addition of a course of NAAD up to 6 months prior to radiation. They observed that NAAD did not confer a therapeutic advantage, but rather added side effects and cost. Furthermore, for the most unfavorable group, there was a higher rate of distant metastasis and more prostate cancer-related deaths. We did not observe a statistical significant benefit on bNED rates with the use of NAAD in none of the RG. 


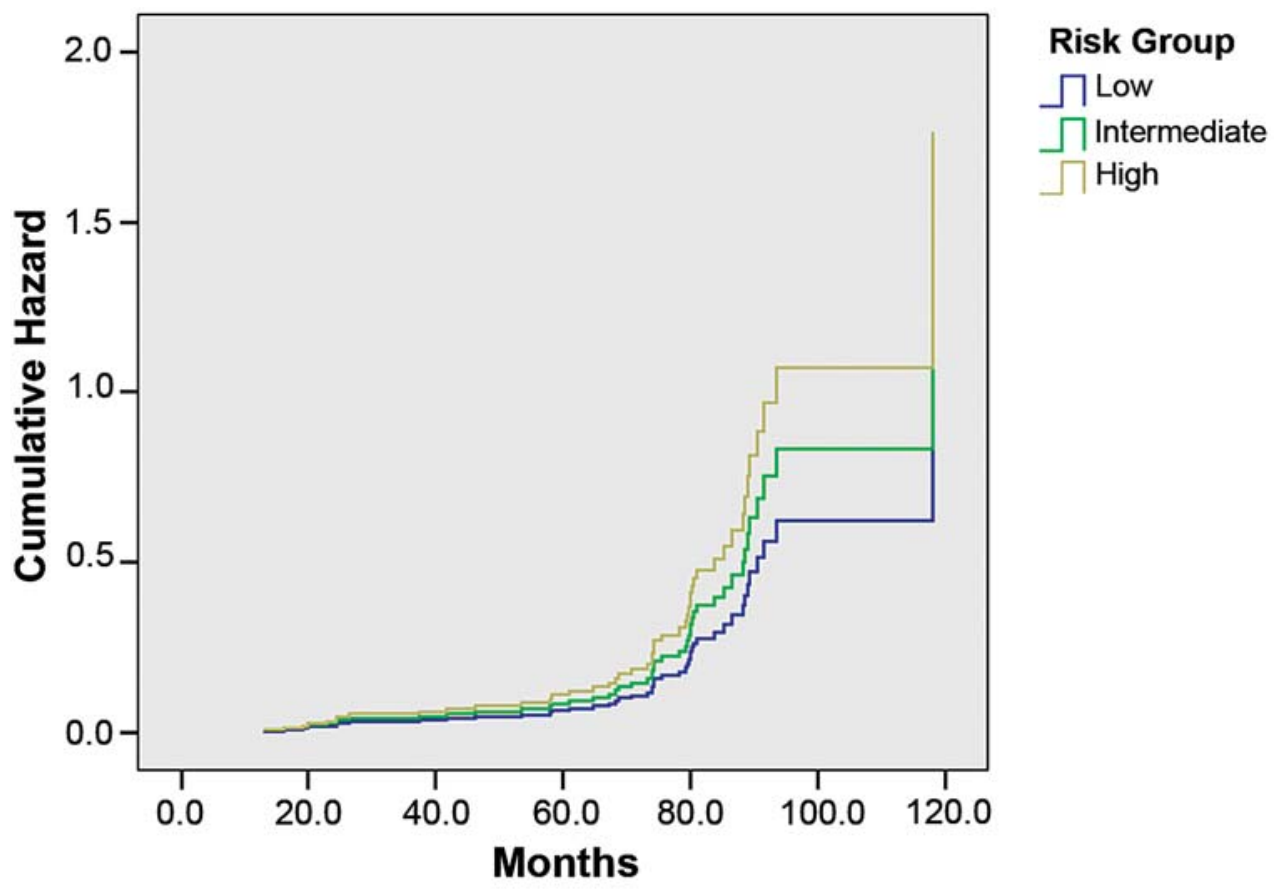

Figure 3 - Hazard functions according to the risk group for biochemical failure.

Aström et al. (21) published their results in terms of bNED for 214 patients consecutively treated from 1988 to 2000 . With a median age of 64 years (50-77) and median follow-up of 4 years (12-165 months), they reported that NAAD was given to 150 patients $(70 \%)$. The overall 5 -year bNED was $82 \%$, and for the LR, IR and HR was $92 \%, 88 \%$ and $61 \%$, respectively.

The use of NAAD and androgen blockage during RT was published by Hsu et al. (22). They reported on their initial 64 patients treated with HDR - 18 Gy given in 3 fractions - to boost RT (45 Gy in 5 weeks). The median follow-up was 50 months (range 25-68 months) and the 4-year actuarial OS and DSS were $98 \%$ and $92 \%$, respectively.

The use of HDR to boost RT is observed worldwide. A Spanish experience using HDR combined with RT was reported by Prada Gomes et al. (23). Between 1998 and 2004, 100 patients considered IR or HR were treated with 46 Gy of RT to the pelvis and 2 HDR brachytherapy fractions (each of $1150 \mathrm{cGy}$ ) at the end of weeks 1 and 3 of a 5-week
RT course. With a median age of 67 years (range 49-78) and median follow-up of 28 months (range: 12-79), the 5-year OS and DSS were $99 \%$ and $87 \%$ respectively.

From Japan, Shigehara et al. (24) evaluated the efficacy of HDR combined with RT. Ninetyseven patients were treated between 1999 and 2003, and 84 patients were analyzed. HDR total dose was 18 Gy given in 3 fractions complemented by RT at a dose of 44 Gy. The 4-year OS and bNED rates were $87.2 \%$ and $82.6 \%$, respectively. Prostate-specific antigen progression-free survival rates of groups with $\mathrm{GS}<7$ and $\mathrm{GS}>$ or $=7$ were $92.8 \%$ and $60.1 \%$, respectively. Oh et al. (25) reported results of $35 \mathrm{HR}$ patients treated between 1995 and 2002. The use of NAAD lasted for a median period of 7 months, followed by concomitant and adjuvant androgen blockage (median time 40 months). The 5-year actuarial bNED rate was $62 \%$. No patients experienced local and/or regional relapse with no distant progression. The 5-year actuarial DSS and OS rates were $89 \%$ and $87 \%$, respectively. 
Preliminary results of an ongoing randomized phase 3 trial with 65 consecutive patients treated with HDR and RT between 1998 and 2004 were published by Chin et al. (26). The median patient age of 67.3 years with the majority of them IR or HR $(92.3 \%)$. With a median follow-up of 3.5 years (range 0.6-5.8), they observed that $2(3 \%)$ patients had died of metastatic disease and another $4(6 \%)$ patients had PSA relapse, giving a 3-year actuarial bNED of $90.8 \%$.

Vargas et al. (27) performed a matched-pair analysis of patients treated with combined RT and HDR from January 1993 to March 2003. A total of 1,432 were evaluated. There were 755 cases identified as having a risk of positive pelvic lymph nodes of more than $15 \%$ using the Roach formula. Of these, 255 cases were treated without pelvic RT and randomly matched by GS, CS and iPSA to 500 cases treated with pelvic $\mathrm{RT}$, resulting in 250 pairs. Based on these results they observed that BF, and OS were not significantly different for patients treated with pelvic radiotherapy. At a median follow-up of 4 years the bNED and OS rates were $78 \%, 86 \%, 89 \%$ and $88 \%$, respectively. In our analysis, the actuarial 5- and 10-year OS were $95.7 \%$ and $90.6 \%$, while the actuarial bNED rates at 5- and 10 -year for the different RG were $91.8 \%$ and $82.3 \%$ for LR, $79.3 \%$ and $67.7 \%$ for IR, and $68.5 \%$ and $41.3 \%$ for HR $(p=0.040)$, respectively.

Our results and others have suggested that bNED is related not only clinical characteristics of patients, as RG and GS, but it is also related to treatment parameters as the total dose given to the prostate, although this could not be proved with statistical significance in this group of patients. In conclusion, we observed that the bNED rates at 3.3 years, reported as 2 years short of the median follow-up, were $91.5 \%$, $90.2 \%$ and $88.5 \%$ for the LR, IR and HR, respectively. To date, in our own experience, HDR associated to RT could be considered a successful approach in the treatment of prostate cancer.

\section{CONFLICT OF INTEREST}

None declared.

\section{REFERENCES}

1. Smith RA, Cokkinides V, Eyre HJ: American Cancer Society guidelines for the early detection of cancer, 2006. CA Cancer J Clin. 2006; 56: 11-25; quiz 4950.

2. Brasil. Ministerio da Saude. Secretaria de Atenção a Saude. Instituto Nacional do Cancer. Coordenação de Prevenção e Vigilancia. Estimativa 2006: Incidência de câncer no Brasil. Rio de Janeiro, INCA. 2005; p. 39. http://www.inca.gov.br/estimativa/2006/versaofinal.pdf

3. Ali AS, Hamdy FC: The spectrum of prostate cancer care: from curative intent to palliation. Curr Urol Rep. 2007; 8: 245-52.

4. Khoo VS: Radiotherapeutic techniques for prostate cancer, dose escalation and brachytherapy. Clin Oncol (R Coll Radiol). 2005; 17: 560-71.

5. Morgan PB, Hanlon AL, Horwitz EM, Buyyounouski MK, Uzzo RG, Pollack A: Radiation dose and late failures in prostate cancer. Int J Radiat Oncol Biol Phys. 2007; 67: 1074-81.

6. Symon Z, Griffith KA, McLaughlin PW, Sullivan M, Sandler HM: Dose escalation for localized prostate cancer: substantial benefit observed with 3D conformal therapy. Int J Radiat Oncol Biol Phys. 2003; 57: 384-90.

7. 7. Vargas CE, Martinez AA, Boike TP, Spencer W, Goldstein N, Gustafson GS, et al.: High-dose irradiation for prostate cancer via a high-dose-rate brachytherapy boost: results of a phase I to II study. Int J Radiat Oncol Biol Phys. 2006; 66: 416-23.

8. 8. Demanes DJ, Rodriguez RR, Schour L, Brandt D, Altieri G: High-dose-rate intensity-modulated brachytherapy with external beam radiotherapy for prostate cancer: California endocurietherapy's 10-year results. Int J Radiat Oncol Biol Phys. 2005; 61: 1306-16.

9. 9. Pellizzon AC, Nadalin W, Salvajoli JV, Fogaroli RC, Novaes PE, Maia MA, et al.: Results of high dose rate afterloading brachytherapy boost to conventional external beam radiation therapy for initial and locally advanced prostate cancer. Radiother Oncol. 2003; 66: 167-72.

10. Pellizzon AC, Salvajoli JV, Maia MA, Ferrigno R, Novaes PE, Fogarolli RC, et al.: Late urinary morbidity with high dose prostate brachytherapy as a boost to conventional external beam radiation therapy for local and locally advanced prostate cancer. J Urol. 2004; 171: 1105-8.

11. Pinkawa M, Fischedick K, Treusacher P, Asadpour B, Gagel B, Piroth MD, et al.: Dose-volume impact in 
high-dose-rate Iridium-192 brachytherapy as a boost to external beam radiotherapy for localized prostate cancer--a phase II study. Radiother Oncol. 2006; 78: 41-6.

12. Soumarová R, Homola L, Stursa M, Perková H: Acute toxicity of conformal high dose interstitial brachytherapy boost in prostate cancer. Neoplasma. 2006; 53: 410-7.

13. Chism DB, Hanlon AL, Horwitz EM, Feigenberg SJ, Pollack A: A comparison of the single and double factor high-risk models for risk assignment of prostate cancer treated with 3D conformal radiotherapy. Int J Radiat Oncol Biol Phys. 2004; 59: 380-5.

14. Roach M 3rd, Hanks G, Thames H Jr, Schellhammer P, Shipley WU, Sokol GH, et al.: Defining biochemical failure following radiotherapy with or without hormonal therapy in men with clinically localized prostate cancer: recommendations of the RTOG-ASTRO Phoenix Consensus Conference. Int J Radiat Oncol Biol Phys. 2006; 65: 965-74.

15. Phan TP, Syed AM, Puthawala A, Sharma A, Khan F: High dose rate brachytherapy as a boost for the treatment of localized prostate cancer. J Urol. 2007; 177: 123-7; discussion 127.

16. Galalae RM, Martinez A, Mate T, Mitchell C, Edmundson $\mathrm{G}$, Nuernberg N, et al.: Long-term outcome by risk factors using conformal high-dose-rate brachytherapy (HDR-BT) boost with or without neoadjuvant androgen suppression for localized prostate cancer. Int $\mathrm{J}$ Radiat Oncol Biol Phys. 2004; 58: 1048-55.

17. Deger S, Boehmer D, Roigas J, Schink T, Wernecke KD, Wiegel T, et al.: High dose rate (HDR) brachytherapy with conformal radiation therapy for localized prostate cancer. Eur Urol. 2005; 47: 441-8.

18. Chin YS, Bullard J, Bryant L, Bownes P, Ostler P, Hoskin PJ: High dose rate iridium-192 brachytherapy as a component of radical radiotherapy for the treatment of localised prostate cancer. Clin Oncol (R Coll Radiol). 2006; 18: 474-9.

19. Yamada Y, Bhatia S, Zaider M, Cohen G, Donat M, Eastham J, et al.: Favorable clinical outcomes of three-dimensional computer-optimized high-doserate prostate brachytherapy in the management of localized prostate cancer. Brachytherapy. 2006; 5: 157-64.

20. Martinez AA, Demanes DJ, Galalae R, Vargas C, Bertermann H, Rodriguez R, et al.: Lack of benefit from a short course of androgen deprivation for unfavorable prostate cancer patients treated with an accelerated hypofractionated regime. Int $\mathrm{J}$ Radiat Oncol Biol Phys. 2005; 62: 1322-31.
21. Aström L, Pedersen D, Mercke C, Holmäng S, Johansson KA: Long-term outcome of high dose rate brachytherapy in radiotherapy of localised prostate cancer. Radiother Oncol. 2005; 74: 157-61.

22. Hsu IC, Cabrera AR, Weinberg V, Speight J, Gottschalk AR, Roach M 3rd, et al.: Combined modality treatment with high-dose-rate brachytherapy boost for locally advanced prostate cancer. Brachytherapy. 2005; 4: 202-6.

23. Prada Gómez PJ, de la Rua Calderón A, Romo Fonseca I, Evia Suárez M, Abascal García JM, Juan Rijo G, et al.: High dose brachytherapy (real time) in patients with intermediate- or high-risk prostate cancer: technical description and preliminary experience. Clin Transl Oncol. 2005; 7: 389-97.

24. Shigehara K, Mizokami A, Komatsu K, Koshida K, Namiki M: Four year clinical statistics of iridium192 high dose rate brachytherapy. Int J Urol. 2006; 13: 116-21.

25. Oh RJ, Yoshioka Y, Tanaka E, Shiomi H, Sumida I, Isohashi F, et al.: High-dose-rate brachytherapy combined with long-term hormonal therapy for high-risk prostate cancer: results of a retrospective analysis. Radiat Med. 2006; 24: 58-64.

26. Chin YS, Bullard J, Bryant L, Bownes P, Ostler P, Hoskin PJ: High dose rate iridium-192 brachytherapy as a component of radical radiotherapy for the treatment of localised prostate cancer. Clin Oncol (R Coll Radiol). 2006; 18: 474-9.

27. Vargas CE, Demanes J, Boike TP, Barnaba MC, Skoolisariyaporn P, Schour L, et al.: Matched-pair analysis of prostate cancer patients with a high risk of positive pelvic lymph nodes treated with and without pelvic RT and high-dose radiation using high dose rate brachytherapy. Am J Clin Oncol. 2006; 29: 451-7.

Accepted after revision:

April 20, 2008

\section{Correspondence address:}

Dr. Antonio Cassio Assis Pellizzon

Rua Prof Antonio Prudente 211

Sao Paulo, SP, 01509-020, Brazil

Fax + $55112189-5101$

E-mail: cassiopellizzon@aol.com 